\title{
Políticas de saúde e alegações de propriedades funcionais e de saúde para alimentos no Brasil
}

\author{
Paulo César Stringheta*1, Tânia Toledo de Oliveira², Ricardo Corrêa Gomes³, Maria da Penha \\ Henriques do Amaral ${ }^{4}$, Antônio Fernandes de Carvalho' ${ }^{1}$, Miriam Aparecida Pinto Vilela ${ }^{4}$
}

\begin{abstract}
${ }^{1}$ Departamento de Tecnologia de Alimentos, Universidade Federal de Viçosa, Viçosa, MG, ${ }^{2}$ Departamento de Bioquímica e Biologia Molecular, Universidade Federal de Viçosa, Viçosa, MG, ${ }^{3}$ Departamento de Administração, Universidade Federal de Viçosa, Viçosa, MG, ${ }^{4}$ Faculdade de Farmácia e Bioquímica, Universidade Federal de Juiz de Fora, Juiz de Fora, MG
\end{abstract}

${ }^{*}$ Correspondência:

P. C. Stringheta

Departamento de Tecnologia de

Alimentos

Universidade Federal de Viçosa 36570-000 - Viçosa - MG, Brasil

E-mail: pstringheta@gmail.com
O trabalho estabelece a relação entre as diretrizes das políticas públicas de saúde brasileiras e os critérios adotados pela Anvisa para aprovação das alegações de propriedades funcionais e, ou, de saúde para alimentos e para substâncias bioativas e probióticos isolados. Comenta a legislação brasileira sobre alimentos com alegações de propriedades funcionais e, ou, de saúde, novos alimentos e substâncias bioativas e probióticos isolados, evidenciando a obrigatoriedade de registro destes produtos junto aos órgãos competentes. Apresenta as diretrizes da Política Nacional de Alimentação e Nutrição, Política Nacional de Promoção da Saúde, Guia Alimentar para a População Brasileira e Estratégia Global sobre Dieta, Atividade Física e Saúde da Organização Mundial de Saúde. Ressalta a convergência das diretrizes destas políticas que objetivam a redução das doenças crônicas não-transmissíveis na população através da promoção da alimentação saudável e da atividade física. Destaca as orientações direcionadas aos governos e à indústria para a implementação dessas medidas. Apresenta critérios de avaliação da base científica das alegações propostos por órgãos internacionais. Objetiva oferecer subsídios para o entendimento da influência da tecnologia de alimentos sobre a saúde pública e para o entendimento dos critérios adotados pela Anvisa, na avaliação das alegações de propriedades funcionais e de saúde.

\section{INTRODUÇÃO}

O termo alimentos funcionais foi inicialmente intro- duzido pelo governo do Japão em meados dos anos 1980, como o resultado de esforços para desenvolver alimentos que possibilitassem a redução dos gastos com saúde públi- 
ca, considerando a elevada expectativa de vida naquele país (Arai, 1996; Hasler, 2002; Araya, Lutz, 2003). O Japão foi pioneiro na formulação do processo de regulamentação específica para os alimentos funcionais. Refere-se aos alimentos processados, similares em aparência aos alimentos convencionais, usados como parte de uma dieta normal e que demonstraram benefícios fisiológicos e, ou, reduziram o risco de doenças crônicas, além de suas funções básicas nutricionais. Conhecidos como Alimentos para Uso Específico de Saúde - Foods for Specified Health Use (FOSHU), estes alimentos trazem um selo de aprovação do Ministério da Saúde e Bem-estar japonês (Hasler, 1998). O princípio foi rapidamente adotado mundialmente (Hasler, 2002). Entretanto, as denominações das alegações ou claims, bem como os critérios para sua aprovação variam de acordo com a regulamentação de cada país ou de blocos econômicos.

Os alimentos funcionais são uma parte importante do bem-estar, no qual também se incluem uma dieta equilibrada e atividade física (IFIC, 2004). O guia alimentar para a população brasileira (Brasil, 2005a) recomenda o estímulo à prática de atividade física, a adoção de uma dieta variada e alerta para não se mistificar os componentes funcionais dos alimentos.

A partir da constatação de que o consumidor tem sido confundido com uma nomenclatura e alegações, claims, de propriedades não demonstradas cientificamente, a tendência do Codex Alimentarius e de vários países foi disciplinar as alegações sobre as propriedades funcionais dos alimentos ou de seus componentes, como também a segurança com base em evidências científicas (Anvisa,1999e, 2004b; Lajolo, 2002; Aggett et al., 2005).

A legislação brasileira não define alimento funcional. Define alegação de propriedade funcional e alegação de propriedade de saúde e estabelece as diretrizes para sua utilização, bem como as condições de registro para os alimentos com alegação de propriedade funcional e, ou, de saúde (Anvisa, 1999b, e, f). Dentre as diretrizes para esse tipo de alimento são permitidas alegações funcionais relacionadas com o papel fisiológico no crescimento, desenvolvimento e funções normais do organismo e, ou, ainda alegações sobre a manutenção geral da saúde e a redução de risco de doenças, em caráter opcional. Não são permitidas alegações que façam referência à cura ou à prevenção de doenças. O alimento ou ingrediente que alegar propriedades funcionais e, ou, de saúde pode, além de funções básicas, quando se tratar de nutriente, produzir efeitos metabólicos e, ou, fisiológicos e, ou, efeitos benéficos à saúde, devendo ser seguro para consumo sem a supervisão médica. Para apresentarem alegações de propriedade funcional e, ou, de saúde, tanto os alimentos como as substân- cias bioativas e probióticos isolados devem ser, obrigatoriamente, registrados junto ao órgão competente. $\mathrm{O}$ conteúdo da propaganda desses produtos não pode ser diferente em seu significado, daquele aprovado para a rotulagem. As alegações devem ainda, estar em consonância com as diretrizes da política pública de saúde (Anvisa, 1999b, e, 2004b, 2005a, Brasil, 1969).

A política pública de saúde do Brasil específica para a área de alimentação e nutrição é a Política Nacional de Alimentação e Nutrição - PNAN (Brasil, 2005b). Ela apresenta interface com a Política Nacional de Promoção da Saúde (Brasil, 2006). O Guia Alimentar para a População Brasileira (Brasil, 2005a) constitui o cumprimento de uma das diretrizes da PNAN. O referido Guia incorporou as recomendações da estratégia global (WHO, 2004) e estabelece as diretrizes para a alimentação saudável e atividade física. As diretrizes destas políticas são utilizadas como critérios para a avaliação das alegações de propriedades funcionais e, ou, de saúde nos alimentos.

O panorama relatado por Santos (2006), em relação ao fluxo de aprovação dos processos de solicitação de registro para alimentos com alegações de propriedades funcionais no período de 1999 a 2004, evidenciou a elevada proporção de processos indeferidos: 214 processos deferidos e 162 indeferidos para alimentos com alegações de propriedades funcionais, 29 processos deferidos e 23 indeferidos para substâncias bioativas e probióticos isolados. Isto demonstra a necessidade de maior compreensão por parte da indústria, dos critérios adotados na avaliação dos processos no que diz respeito aos termos utilizados na legislação (Anvisa, 2004b) "em consonância com as diretrizes da política pública de saúde", "à luz dos conhecimentos científicos atualizados" e "alimentos de consumo ocasional".

Este trabalho utilizou a metodologia de pesquisa documental objetivando reunir informações relacionadas à legislação brasileira, às políticas públicas de saúde e alimentação e à estratégia global sobre dieta, atividade física e saúde, da Organização Mundial de Saúde, considerando que o conhecimento do significado dos termos utilizados nas regulamentações e nas políticas públicas, bem como o conhecimento do papel dos diversos agentes, stakeholders, que influenciam nessas políticas são fundamentais. Desta forma, espera-se contribuir para que os projetos de desenvolvimento de produtos possam ser racionalizados, resultando em benefícios para os fabricantes, órgãos de saúde pública e para os consumidores.

\section{LEGISLAÇÃO BRASILEIRA}

Segundo o Decreto n 3029, de 16 de abril de 1999 
(Brasil, 1999a), alterado pelo decreto ${ }^{\circ} 3571$, de 21 de agosto de 2000 (Brasil, 2000), são competências da Agência Nacional de Vigilância Sanitária: estabelecer normas, acompanhar e executar as políticas, as diretrizes e as ações de vigilância sanitária; conceder registros de produtos, segundo as normas de sua área de atuação; controlar, fiscalizar e acompanhar, sob o prisma da legislação sanitária, a propaganda e a publicidade de produtos submetidos ao regime de vigilância sanitária.

No Brasil, desde 1990 existiam pedidos de registro de diversos produtos até então não reconhecidos como alimentos, dentro do conceito tradicional de alimento. Somente em 1999, a Anvisa aprovou a regulamentação que trata das diretrizes básicas para avaliação do risco e segurança dos alimentos, Resolução n¹7/99 (Anvisa, 1999d), dos procedimentos para registro de alimentos e, ou, novos ingredientes, Resolução n¹6/99 (Anvisa, 1999c), das diretrizes básicas para análise e comprovação de alegação de propriedade funcional e, ou, de saúde alegadas em rotulagem de alimentos, Resolução no 18/99 (Anvisa,1999e) e Portaria 398/99 (Anvisa,1999b) e dos procedimentos para registro de alimento com alegação de propriedades funcionais e, ou, de saúde, Resolução n 19/99 (Anvisa, 1999f). Estas categorias de alimentos vêm sendo introduzidas para consumo livre pela população (Carvalho, 2004).

Com a mudança no enfoque de análise dos alimentos, que passou a considerar o critério de risco, a Vigilância Sanitária aprovou a Portaria n¹5/99 (Anvisa, 1999a), que constituiu a Comissão de Assessoramento Tecnocientífico em Alimentos Funcionais e Novos Alimentos CTCAF, com a função de subsidiar a Diretoria de Alimentos e Toxicologia nas decisões relacionadas a esse tema. A denominação da CTCAF foi alterada pela Portaria 386/ 2005 (Anvisa, 2005b) para Comissão de Assessoramento Tecnocientífico em Alimentos com Alegação de Propriedade Funcional e, ou, de Saúde e Novos Alimentos.

Segundo a Anvisa (1999c, 2006), "alimentos e, ou, novos ingredientes" são os alimentos ou substâncias sem histórico de consumo no País, ou alimentos com substâncias já consumidas, e que, entretanto venham a ser adicionadas ou utilizadas em níveis muito superiores aos atualmente observados nos alimentos utilizados na dieta regular. Os alimentos que vierem a ser consumidos nas formas de cápsulas, comprimidos ou outras formas farmacêuticas, e que não apresentem alegação de propriedade funcional ou de saúde cientificamente comprovada devem trazer no rótulo a afirmação "O Ministério da Saúde adverte: Não existem evidências científicas comprovadas de que este alimento previna, trate ou cure doenças".

Outras regulamentações como as Resoluções $n^{\circ} 22$ e $n^{\circ} 23$, Resolução RDC $n^{\circ} 2$, Informes Técnicos $n^{\circ} 9$ e $n^{\circ}$
19 (Anvisa, 2000a, b, 2002, 2004b, 2006) foram aprovadas, além das regulamentações horizontais, como a Resolução $\mathrm{n}^{\circ} 278$ (Anvisa, 2005c) que se aplicam para todos os tipos de alimentos.

$A \mathrm{RDC} \mathrm{n}^{\circ}$ 2/2002 (Anvisa, 2002) se aplica às diretrizes a serem adotadas para a avaliação de segurança, registro e comercialização de substâncias bioativas e probióticos isolados com alegação de propriedades funcional e, ou, de saúde, apresentadas como formas farmacêuticas (cápsulas, comprimidos, tabletes, pós, granulados, pastilhas, suspensões e soluções). Os produtos são classificados em: carotenóides, fitoesteróis, flavonóides, fosfolípideos, organossulfurados, polifenóis e probióticos. Uma vez aprovadas, as alegações propostas pelo fabricante são de uso obrigatório.

O registro na Anvisa é obrigatório tanto para as substâncias bioativas e probióticos isolados como para alimentos com alegação de propriedade funcional e, ou, de saúde e para os alimentos novos e novos ingredientes, produzidos no Brasil ou importados (Anvisa, 2000a, b, 2005c). Mesmo para os produtos de origem animal, de competência do Ministério da Agricultura, o processo referente à comprovação das alegações deve ser encaminhado pelo referido Ministério à Anvisa para análise.

O Informe Técnico n ${ }^{\circ} 9$ de maio de 2004 (Anvisa, 2004b) foi elaborado a partir da constatação de que a aplicação do item 3.3 da resolução n 18/99 "para os nutrientes com funções plenamente reconhecidas pela comunidade científica não será necessária a demonstração de eficácia ou análise da mesma para alegação funcional na rotulagem" (Anvisa, 1999e) possibilitava situações que contrariam as Diretrizes das Políticas Públicas de Saúde, e da observação do aumento da utilização de alegações em rótulos de produtos dispensados da obrigatoriedade de registro no comércio (Anvisa, 2004b). De acordo com o referido informe, os seguintes critérios devem ser cumpridos para aprovação das alegações para nutrientes com função plenamente reconhecidas pela comunidade científica:

- estarem relacionadas a nutrientes intrínsecos ao produto, os quais devem estar presentes pelo menos na quantidade estabelecida para o atributo "fonte" conforme a regulamentação sobre Informação Nutricional Complementar - Portaria nº 27/98 (Brasil,1998);

- serem específicas quanto à função do nutriente objeto da alegação;

- estarem vinculadas ao alimento de consumo habitual da população, o qual não deve ser de consumo ocasional e nem estar apresentado em cápsulas, comprimidos, tabletes ou outras formas farmacêuticas.

$\mathrm{O}$ atendimento aos critérios estabelecidos para o uso 
das alegações previstas no item 3.3 da Resolução ANVS/ MS no 18/99 (Anvisa, 1999e), de responsabilidade da empresa, dispensa o envio de documentação para avaliação técnica, ressaltando que as alegações não podem fazer referência a prevenção, tratamento e cura de doenças. Entretanto, a dispensa refere-se apenas à necessidade de comprovação das alegações - Item 4.1.1.9 da RDC 18/99 (Anvisa, 1999e) e não ao registro e aos demais itens do relatório técnico-científico (item 4.1.1). Os alimentos adicionados de nutrientes essenciais, que façam alegações de propriedades funcionais, devem ser encaminhados para avaliação caso a caso.

Em relação à expressão "não deve ser de consumo ocasional" (Anvisa, 2004b), segundo a RDC n 359/2003 (Anvisa, 2003), são considerados de consumo ocasional os produtos: frutas inteiras em conserva para adornos (cerejas maraschino, framboesa); balas, pirulitos e pastilhas; goma de mascar; chocolates, bombons e similares; confeitos de chocolate e drageados em geral; sorvetes de massa; sorvetes individuais; barra de cereais com mais de $10 \%$ de gorduras, torrones, pé-de-moleque e paçoca; bebidas não-alcoólicas, carbonatadas ou não (chás, bebidas à base de soja e refrigerantes); pós para preparo de refresco; biscoito doce, com ou sem recheio; brownies e alfajores; frutas cristalizadas; panettone; bolo com frutas; bolos e similares com recheio e cobertura; snacks à base de cereais e farinhas para petisco; mistura para preparo de docinho, cobertura para bolos, tortas e sorvetes, entre outros.

De acordo com a Anvisa (2005a), após cinco anos foram reavaliados os produtos com alegações de propriedades funcionais e, ou, de saúde aprovados desde o ano de 1999. Utilizaram-se como base os conhecimentos científicos atualizados, bem como relatos e pesquisas que demonstraram as dificuldades encontradas pelos consumidores em entender o verdadeiro significado da característica anunciada para determinados produtos contendo alegações. A revisão considerou como pressupostos a necessidade das alegações estarem de acordo com as políticas do Ministério da Saúde e serem de fácil compreensão pelos consumidores, além de cumprir com o estabelecido pelas Resoluções nº 17/99, 18/99 e 19/99. Os seguintes produtos tiveram as suas alegações modificadas, com o intuito de aprimorar o entendimento dos consumidores quanto às propriedades destes alimentos: ácidos graxos da família ômega 3, carotenóides (licopeno e luteína), fibras alimentares (fibras alimentares, beta-glucana, frutooligossacarídeos, inulina, lactulose, Psillinum ou Psillium, quitosana), fitoesteróis, probióticos (Lactobacillus acidophilus, L. casei shirota, L. casei var. rhammosus, $L$. casei var. defensis, L. delbrueckii subspécie bulgaricus,
Bifidobacterium bifidum, B. lactis, B. longum, Streptococcus salivarius subespécie thermophillus) e proteína de soja. As alegações anteriormente aprovadas, relacionadas à cafeína, ao sorbitol, ao xilitol, ao manitol, ao estearato de sódio, ao bicarbonato de sódio, ao ômega 6, aos ácidos graxos monoinsaturados e poliinsaturados (em óleos vegetais) e ao composto líquido pronto para consumo, não foram mais permitidas. As empresas deveriam adequar os dizeres de rotulagem, seguindo este novo formato das alegações até 30 de janeiro de 2006 ou no prazo negociado junto às vigilâncias regionais para o esgotamento das embalagens dos produtos (Anvisa, 2005a).

Segundo Santos (2006), a situação atual no Brasil, após a reavaliação das alegações em 2004, era a seguinte: nenhuma alegação de saúde foi aprovada. Estão aprovadas 14 alegações de propriedades funcionais genéricas com linguagem padronizada e 25 tipos de substâncias ou microrganismos com alegação de propriedade funcional. As alegações sobre nutrientes com função plenamente estabelecida encontravam-se em discussão (Santos, 2006; Lajolo, 2006; Anvisa, 2005a).

A propaganda e a publicidade destes produtos são fiscalizadas pela Anvisa, através da Gerência de Monitoração e Fiscalização da Propaganda, de Publicidade, de Promoção e de Informação de Produtos Sujeitos à Vigilância Sanitária - GPROP (Anvisa, 2004a). Qualquer folheto de informação ao consumidor, que componha a embalagem do produto, ou seja, um instrumento de divulgação do mesmo, não poderá veicular informação alusiva as suas propriedades que não sejam aquelas aprovadas pelo órgão competente da Anvisa para constar em sua rotulagem, conforme estabelece o Artigo 23 do DecretoLei $n^{\circ}$ 986/69 (Brasil, 1969; Anvisa, 2005a).

\section{CRITÉRIOS PARA AVALIAÇÃO DA BASE CIENTÍFICA DAS ALEGAÇÕOES}

A Organização Mundial da Saúde - OMS, em conjunto com a Organização para Agricultura e Alimentação, WHO/FAO (WHO, 2003) publicou algumas recomendações quanto ao estilo de vida, dieta e consumo de alimentos, sugerindo níveis de evidência científica para o risco de desenvolvimento de doenças crônicas não-transmissíveis. A prática dietética ou nutricional recomendada deve modificar o risco atribuível de uma doença naquela população. A classificação da força da evidência da WHO/FAO (WHO, 2003) para as recomendações na prática clínica para prevenção de doenças, de acordo com a qualidade, quantidade e resultados dos estudos disponíveis, é estabelecida como evidência convincente, provável, possível e insuficiente. A força de evidência que relaciona os fatores de dieta e de 
TABELA I - Sumário da força de evidência para obesidade, diabetes tipo 2, doença cardiovascular (CVD) e câncer ${ }^{\text {a }}$

\begin{tabular}{|c|c|c|c|c|}
\hline & Obesidade & Diabetes tipo 2 & CVD & Câncer \\
\hline Ingestão elevada de alimentos altamente energéticos & $\mathrm{C} \uparrow$ & & & \\
\hline Ácidos graxos trans & & & $\mathrm{C} \uparrow$ & \\
\hline Peixe e óleo de peixe (EPA e DHA) & & & $C \downarrow$ & \\
\hline Alta ingestão de fibra dietética (NSP) & $C \downarrow$ & $\mathrm{P} \downarrow$ & $\mathrm{P} \downarrow$ & \\
\hline Alta ingestão de sódio & & & $\mathrm{C} \uparrow$ & \\
\hline Frutas (incluindo frutas vermelhas) e vegetais & $C \downarrow^{k}$ & $\mathrm{P} \downarrow^{k}$ & $C \downarrow$ & $P \downarrow 1$ \\
\hline Sobrepeso e obesidade & & $\mathrm{C} \uparrow$ & $\mathrm{C} \uparrow$ & $\mathrm{C} \uparrow \mathrm{s}$ \\
\hline Atividade física regular & $C \downarrow$ & $\mathrm{C} \downarrow$ & $C \downarrow$ & $\mathrm{C} \downarrow$ \\
\hline
\end{tabular}

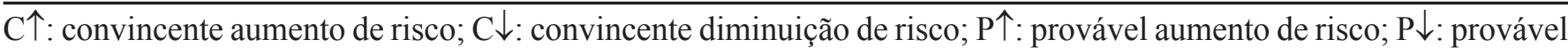
diminuição de risco; EPA: ácido eicosapentanóico; DHA: ácido docosapentanóico; NSP: polissacarídeos não-amiláceos.

${ }^{a}$ Somente evidências Convincente $(\mathrm{C})$ e prováveis $(\mathrm{P})$ estão incluídas nesta tabela sumária.

${ }^{\mathrm{k}}$ Baseado nas contribuições de frutas e vegetais com polissacarídeos não-amiláceos (fibras dietéticas).

${ }^{1}$ Para câncer da cavidade oral, esôfago e colo-retal.

s Para câncer de esôfago, colo-retal, mama (em mulheres pós-menopausa), endométrio e rim.

Fonte: adaptada de WHO (2003).

estilo de vida com o risco de desenvolvimento de obesidade, diabetes tipo 2, doenças cardiovasculares (CVD) e câncer, classificadas de acordo com as categorias citadas acima, estão resumidas na Tabela I.

\section{POLÍTICAS GERAIS: ALIMENTOS E PROMOÇÃO DA SAÚDE}

Existe um acentuado interesse mundial para melhorar a qualidade da nutrição e reduzir os gastos com saúde por meio da prevenção de doenças crônicas, da melhoria da qualidade e da expectativa de vida ativa. As políticas de saúde brasileiras referentes a alimentação e nutrição apresentam esta tendência e seguem as recomendações da estratégia global sobre dieta atividade física e saúde, publicada pela Organização Mundial da Saúde (WHO, 2004). A estratégia recomenda aos países-membros a adoção de políticas que estimulem a alimentação saudável e a prática de atividade física, como forma de diminuir a ocorrência das doenças crônicas não-transmissíveis causadas pela alimentação não-saudável e por estilos de vida sedentários. As políticas nacionais e internacionais que influenciam na aprovação das alegações para alimentos estão apresentadas, a seguir, em ordem cronológica.

\section{Política Nacional de Alimentação e Nutrição - PNAN (Brasil, 1999b, 2005b)}

A PNAN foi aprovada pelo Conselho Nacional de Saúde e pela Portaria ${ }^{\circ} 710$ do Ministério da Saúde, de 10 de junho de 1999 (Brasil, 1999b). Tem como propósito a garantia da qualidade dos alimentos colocados para consumo no País, a promoção de práticas alimentares saudáveis e a prevenção e o controle dos distúrbios nutricionais, bem como o estímulo às ações intersetoriais que propiciem o acesso universal aos alimentos. Ela integra a Política Nacional de Saúde e apresenta interface com a Política Nacional de Promoção da Saúde, inserindo-se, no contexto da Segurança Alimentar e Nutricional (Brasil, 2005b). A Figura 1 ilustra as interfaces da Política Nacional de Alimentação e Nutrição e Política Nacional de Promoção da Saúde com a Política Nacional de Saúde.

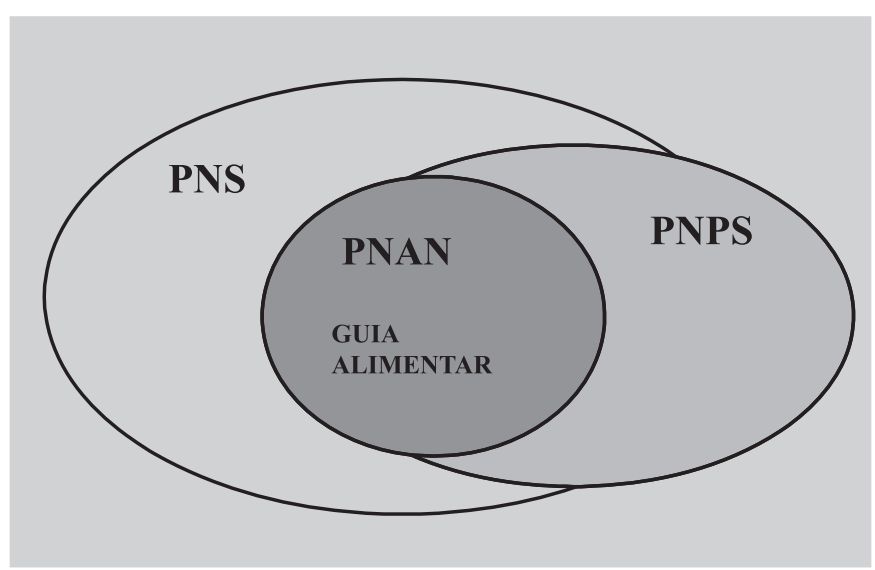

FIGURA 1 - Interfaces das Políticas Nacionais Brasileiras de Saúde (PNS), de Alimentação e Nutrição (PNAN), Guia Alimentar para a População Brasileira (GAPB), e de Promoção da Saúde (PNPS) - Fonte: adaptada de Oliveira (2006). 
A Política Nacional de Alimentação e Nutrição (Brasil, 2005b) faz algumas considerações sobre a realidade nacional, das quais se destacam:

- A adoção do conceito de Segurança Alimentar e Nutricional em âmbito mundial e no Brasil facilitaram a compreensão do papel do setor saúde no tocante à alimentação e nutrição, reconhecidas como elementos essenciais para a promoção, proteção e recuperação da saúde.

- A obesidade na população brasileira está se tornando bem mais freqüente do que a própria desnutrição infantil, sinalizando um processo de transição epidemiológica que deve ser devidamente valorizado no plano de saúde coletiva. As doenças cardiovasculares, que representam a principal causa de morte e de incapacidade na vida adulta e na velhice e são responsáveis, no Brasil, por $34 \%$ de todas as causas de óbito, estão relacionadas, em grande parte, com a obesidade e com práticas alimentares e estilos de vida inadequados.

- Os hábitos alimentares inapropriados constituem um grande desafio. A cultura popular ainda preserva tradições e práticas alimentares errôneas sobre o valor nutritivo, propriedades terapêuticas, indicações ou interdições de alimentos ou de suas combinações. Ressalta-se, de outra parte, a multiplicação do comércio de fast food e o crescente uso de alimentos pré-cozidos ou de cozimento rápido.

- Constata-se que é bastante complexa a situação da alimentação e nutrição no Brasil, onde coexistem problemas típicos de sociedades subdesenvolvidas e de países desenvolvidos (Brasil, 2005b).

Para o alcance de seu propósito a PNAN apresenta sete diretrizes: 1. Estímulo às ações intersetoriais com vistas ao acesso universal aos alimentos; 2. Garantia da segurança e da qualidade dos alimentos e da prestação de serviços nesse contexto; 3 . Monitoramento da situação alimentar e nutricional do País; 4. Promoção de práticas alimentares saudáveis; 5 . Prevenção e controle dos distúrbios nutricionais e doenças associadas à alimentação e nutrição; 6. Desenvolvimento de linhas de investigação; 7 . Desenvolvimento e capacitação de recursos humanos. Destacam-se aqui as diretrizes 2, 4 e 5:

Diretriz 2. Garantia da segurança e da qualidade dos alimentos e da prestação de serviços nesse contexto: o redirecionamento e o fortalecimento das ações de vigilância sanitária serão focos de atenção especial, com vistas à proteção da saúde do consumidor. No âmbito do Sistema Nacional de Vigilância Sanitária, deverá ser fortalecido o componente relativo a alimentos e serviços de alimentação, mediante a revisão ou adequação de normas, enfatizando aquelas relacionadas à prevenção de agravos à saúde.

Diretriz 4. Promoção de práticas alimentares saudáveis: a promoção de práticas alimentares saudáveis, que se inicia com o incentivo ao aleitamento materno, está inserida no contexto da adoção de estilos de vida saudáveis. Nesse sentido, ênfase será dada à socialização do conhecimento sobre os alimentos e o processo de alimentação, bem como acerca da prevenção dos problemas nutricionais, desde a desnutrição - incluindo as carências específicas até a obesidade. Deverá ser concedida ênfase particular à orientação quanto à prevenção de doenças crônicas nãotransmissíveis, tais como as cardiovasculares e a diabetes mellitus, e à adoção de hábitos alimentares apropriados por seus portadores.

Serão contempladas iniciativas específicas dirigidas ao aleitamento materno e à adoção de medidas voltadas a disciplinar a publicidade de produtos alimentícios infantis, sobretudo em parceria com as entidades da sociedade civil e do setor produtivo. Serão implementadas iniciativas que possibilitem o acompanhamento e o monitoramento de práticas de marketing sob os critérios e interesses de uma vida efetivamente saudável, sendo objeto de atenção as questões relacionadas ao sobrepeso e suas implicações.

Diretriz 5. Prevenção e controle dos distúrbios nutricionais e doenças associadas à alimentação e nutrição: no grupo das enfermidades crônicas não-transmissíveis, as medidas estarão voltadas à promoção da saúde e ao controle dos desvios alimentares e nutricionais, por constituírem as condutas mais eficazes para prevenir sua instalação e evolução. Deverão ser consolidados os esforços destinados a ampliar a duração do aleitamento materno, de modo que a prática da amamentação exclusiva se estenda até aos seis meses de idade e o aleitamento materno combinado com a introdução de alimentos adequados se prolongue até o segundo ano de vida.

Algumas definições relevantes de acordo com a PNAN (Brasil, 2005b):

Hábitos alimentares saudáveis: compõem-se de práticas alimentares saudáveis e de segurança e qualidade dos alimentos.

Práticas alimentares saudáveis: usos, hábitos e costumes que definem padrões de consumo alimentar de acordo com os conhecimentos científicos e técnicas de uma boa alimentação.

Segurança e qualidade dos alimentos: trata, em vigilância sanitária, dos atributos referentes à inocuidade dos alimentos e seu valor nutritivo. Ver também práticas alimentares saudáveis.

Segurança Alimentar: garantia de que as famílias te- 
nham acesso físico e econômico regular e permanente a conjunto básico de alimentos em quantidade e qualidade significativas para atender os requerimentos nutricionais.

Segurança Alimentar e Nutricional: acrescenta-se à definição anterior, o conceito de que, além do acesso e consumo, o organismo deve dispor de condições fisiológicas adequadas para o aproveitamento dos alimentos, ou seja, para uma boa digestão, absorção e metabolismo de nutrientes.

\section{Estratégia global para dieta, atividade física e saúde - OMS (WHO, 2004)}

A estratégia global para dieta, atividade física e saúde foi aprovada pela $57^{\mathrm{a}}$ Assembléia da Organização Mundial da Saúde, atendendo à solicitação dos paísesmembros (WHO, 2004). As doenças não-transmissíveis impõem uma carga econômica significativa sobre os sistemas de saúde existentes e infringem altos custos na sociedade. A Comissão sobre Macroeconomia e Saúde da OMS demonstrou o efeito desordenador da doença sobre o desenvolvimento e a importância de investimentos em saúde para o desenvolvimento econômico (WHO, 2001). Os programas que objetivam a promoção de dietas saudáveis e atividade física para a prevenção de doenças são instrumentos chave nas políticas que visam alcançar as metas de desenvolvimento. A estratégia global é dirigida aos dois principais riscos para doenças não-transmissíveis: a dieta e a atividade física, enquanto outro estudo, que se encontra em andamento, enfoca as doenças carenciais (WHO, 2004).

A carga de doenças não-transmissíveis tem aumentado rapidamente no mundo. Em 2001, as doenças nãotransmissíveis eram quase $60 \%$ das 56 milhões de mortes anuais e $47 \%$ da carga global de doenças. Em vista desta realidade e prevendo o crescimento futuro, a prevenção das doenças não-transmissíveis representa o maior desafio para a saúde pública global (WHO, 2002).

As dietas não saudáveis e a inatividade física estão entre as causas das principais doenças não-transmissíveis, incluindo as doenças cardiovasculares, diabetes tipo $2 \mathrm{e}$ certos tipos de câncer, e contribuem substancialmente para a carga global de doenças, mortes e incapacidades.

Em todos os países estudados, os fatores que aumentaram os riscos das doenças não-transmissíveis foram o consumo elevado de alimentos densos em energia, pobres em nutrientes e ricos em gorduras, açúcar e sal; níveis reduzidos de atividade física em casa, na escola, no trabalho e para recreação e transporte e o uso de tabaco (WHO, 2004).

A estratégia reconhece que os governos têm um papel central, em cooperação com outros agentes, stakeholders, para criarem um ambiente que estimule e encoraje mudanças de comportamento de indivíduos, famílias e comunidades, para fazerem escolhas positivas de melhoria de vida relacionadas com padrões de dieta e atividade física.

O objetivo geral da estratégia global sobre dieta, atividade física e saúde é promover e proteger a saúde pelo direcionamento do desenvolvimento de uma capacitação do ambiente, para sustentar as ações em níveis individuais, comunitários, nacionais e globais que, quando tomadas juntas, levarão a redução das taxas de doenças e de mortes relacionadas a dietas não saudáveis e inatividade física. Os principais objetivos específicos são:

1- reduzir os fatores de risco para doenças crônicas não-transmissíveis (DCNT) através da ação essencial em saúde pública, promoção da saúde e medidas preventivas;

2- aumentar a atenção e o conhecimento sobre alimentação e atividade física;

3- encorajar o desenvolvimento, fortalecimento e implementação de políticas e planos de ação em nível global, regional, nacional e comunitário que sejam sustentáveis, incluindo a sociedade civil, o setor privado e a mídia;

Em relação à dieta, deve-se recomendar para indivíduos e populações: manter o equilíbrio energético e o peso saudável; limitar a ingestão energética procedente de gorduras, substituir as gorduras saturadas por insaturadas e eliminar as gorduras trans (hidrogenadas); aumentar o consumo de frutas, legumes e verduras, grãos integrais e nozes; limitar a ingestão de açúcar livre; limitar a ingestão de sal (sódio) de toda procedência e consumir sal iodado (WHO, 2004).

Com respeito à atividade física, a Estratégia Global recomenda pelo menos 30 minutos de atividade física, regular, intensa ou moderada quase todos os dias, para reduzir o risco de doenças cardiovasculares, diabetes, câncer de colón e de mama e melhorar o estado funcional nas diferentes fases da vida, especialmente na fase adulta e idosa (WHO, 2004).

\section{Responsabilidades}

Para alcançar as mudanças nos hábitos alimentares e padrões de atividade física é necessário o esforço combinado de muitos agentes públicos e privados (Organização Mundial da Saúde, governos, parceiros internacionais, sociedade civil e organizações não-governamentais, setor privado) durante várias décadas. Dentre as responsabilidades e recomendações, destacam-se:

a) Para os Estados-Membros

Os governos devem considerar ações que resultarão em fornecimento de informação exata e balanceada para os consumidores para capacitá-los a fazer escolhas saudá- 
veis. As informações para os consumidores devem ser adequadas para níveis de alfabetização, barreiras de comunicação, culturas locais e entendidas por todos os segmentos da população.

- Marketing, propaganda, patrocínio e promoção: a propaganda de alimentos afeta as escolhas dos alimentos e influencia os hábitos alimentares. A publicidade dos alimentos e bebidas não deveria explorar a credulidade e a inexperiência infantis. Mensagens que encorajam práticas dietéticas não saudáveis ou a inatividade física devem ser desencorajadas, enquanto que mensagens positivas e saudáveis devem ser encorajadas.

- Rotulagem: os consumidores necessitam de informação exata, padronizada e compreensível sobre o conteúdo dos alimentos para fazerem escolhas saudáveis.

- Alegações de saúde: como o interesse em saúde dos consumidores está aumentando, o uso de mensagens relacionadas à saúde pelos fabricantes está aumentando constantemente. Tais mensagens não devem confundir o público sobre os benefícios ou riscos nutricionais.

- Promoção de produtos alimentícios consistente com uma dieta saudável: os governos poderiam considerar medidas para estimular a redução do conteúdo de sal nos alimentos processados, do uso de gorduras hidrogenadas e do conteúdo de açúcar em bebidas e guloseimas.

b) Para o setor privado

A indústria de alimentos como um dos representantes do setor privado pode ser um agente significativo na promoção de dietas saudáveis e atividade física. Iniciativas da indústria de alimentos para diminuir o conteúdo de gordura, açúcar e sal nos alimentos processados e o tamanho das porções, para aumentar a introdução de escolhas nutritivas, saudáveis e inovadoras, e rever as práticas atuais de marketing, poderiam acelerar os ganhos de saúde mundial.

\section{Recomendações específicas para a indústria de} alimentos

- Promover dietas saudáveis e atividade física em consonância com as diretrizes e padrões nacionais e internacionais e com os objetivos gerais da estratégia global; limitar os níveis de gorduras saturadas, ácidos graxos trans, açúcares livres e sal nos produtos existentes.

- Continuar a desenvolver e fornecer escolhas nutritivas, saudáveis e de custo acessível para os consumidores.

- Considerar a introdução de produtos com melhor valor nutricional.
- Fornecer aos consumidores informação adequada e compreensível sobre nutrição e os produtos.

- Praticar o marketing responsável, que apóia a estratégia, particularmente com respeito à promoção e "marketing" de alimentos com elevado conteúdo de gorduras saturadas, ácidos graxos trans, açúcares livres ou sal, especialmente para crianças.

- Emitir rótulos simples, claros e consistentes e alegações de saúde fundamentadas em evidências, que ajudem os consumidores a fazerem escolhas conscientes e saudáveis com relação ao valor nutritivo dos alimentos.

- Fornecer informação para as autoridades nacionais sobre a composição dos alimentos.

De acordo com a WHO (2004), a implementação desta estratégia por todos os agentes envolvidos contribuirá para a melhoria sustentável da saúde humana.

\section{Guia alimentar para a população brasileira (Brasil, 2005a)}

A edição das primeiras diretrizes alimentares oficiais do Brasil fez parte da estratégia de implementação da Política Nacional de Alimentação e Nutrição, integrante da Política Nacional de Saúde (Brasil, 2005b). Consolidase como elemento concreto de identidade brasileira para implementação das recomendações preconizadas pela Organização Mundial da Saúde (WHO, 2004). O Guia foi publicado em um momento da história do conhecimento, quando as evidências científicas acumuladas até o momento apontam de forma inequívoca e consistente, o impacto da alimentação saudável na prevenção das mortes prematuras causadas pelas doenças cardíacas e câncer no Brasil e em grande parte dos países do mundo (WHO, 2003; Brasil, 2005a).

O Guia (Brasil, 2005a) tem como propósito contribuir para a orientação de práticas alimentares que visem à promoção da saúde e à prevenção de doenças relacionadas à alimentação:

- desnutrição e deficiências por micronutrientes como a anemia ferropriva, a hipovitaminose A e distúrbios por carência de iodo, que ainda permanecem sendo problemas de saúde pública no País e

- doenças crônicas não-transmissíveis - DCNT (diabetes, obesidade, hipertensão arterial, doenças cardiovasculares e alguns tipos de câncer).

A edição se baseia na noção de que a alimentação se dá em função do consumo de alimentos e não de nutrientes, ou seja, uma alimentação saudável deve ser baseada em práticas alimentares que tenham significado social e cultural. Considera que todas as características como o 
gosto, cor, forma, aroma e textura dos alimentos precisam ser considerados na abordagem nutricional. $\mathrm{O}$ alimento como fonte de prazer e identidade cultural e familiar é também uma abordagem necessária para a promoção da saúde. A seguir destacam-se algumas considerações contidas no Guia (Brasil, 2005a) e alguns aspectos da Diretriz 1 - Os alimentos saudáveis e as refeições e da Diretriz Especial 1 - Atividade Física.

Nas últimas duas ou três gerações, a sociedade brasileira tornou-se predominantemente urbana. As doenças crônicas do total da população no País aumentaram de $34,4 \%$, em 1979, para 48,3\%, em 2003. Conseqüentemente, os serviços e as políticas públicas precisam responder a estas transformações e à complexidade de suas manifestações na saúde (Brasil, 2005a).

\section{A transição epidemiológica brasileira}

Com a urbanização da população os padrões de trabalho e lazer mudaram para menor gasto energético. Por outro lado, o aumento no consumo de alimentos processados, ricos em gordura, açúcar e sal, associado ao menor gasto energético diário devido à redução da atividade física, explicam as tendências crescentes de sobrepeso e obesidade na população brasileira e das DCNT associadas (Brasil, 2005a).

De acordo com Brasil (2005a), até poucos anos, os principais desafios em saúde pública relacionados à alimentação eram a desnutrição e as deficiências de micronutrientes entre crianças, bem como as doenças infecciosas, principalmente na infância e adolescência. No entanto, a evolução das doenças crônicas não-transmissíveis (DCNT) coloca-se como desafio adicional à segurança alimentar e nutricional, o qual deve ser conjugado com os esforços para a reversão da prevalência da desnutrição infantil e no controle e prevenção das deficiências de micronutrientes, que ainda acometem milhões de indivíduos de diferentes cursos da vida.

\section{Modos de vida saudáveis}

Evidências científicas recentes mostram que a saúde pode estar muito mais relacionada ao modo de viver das pessoas do que a idéia, anteriormente hegemônica, da sua determinação genética e biológica. Na abordagem da promoção de modos de vida saudáveis, identificam-se duas dimensões: aquela que se propõe a estimular e incentivar práticas saudáveis, a alimentação saudável e a atividade física regular e, outra, que objetiva a inibição de hábitos e práticas prejudiciais à saúde como o consumo de tabaco e de álcool. A alimentação saudável tem início com a prática do aleitamento materno e se prolonga pela vida com adoção de bons hábitos alimentares. Assim, a nutri- ção adequada de gestantes e crianças deve ser entendida e enfatizada como elemento estratégico de ação, com vistas à promoção da saúde também na vida adulta.

\section{Alimentação saudável: algumas considerações}

Em geral, as escolhas alimentares são determinadas não tanto pela preferência e pelos hábitos, mas muito mais pelo sistema de produção e de abastecimento de alimentos (Brasil, 2005a). Se esses sistemas produzem alimentos que são inadequados ou inseguros e que aumentam os ris$\cos$ de doenças, eles precisam ser mudados. O Estado, por intermédio de suas políticas públicas, tem a responsabilidade de fomentar mudanças sócio-ambientais, em nível coletivo, para favorecer as escolhas saudáveis em nível individual ou familiar. Assim, é pressuposto da promoção da alimentação saudável ampliar e fomentar a autonomia decisória dos indivíduos e grupos, por meio do acesso à informação para a escolha e adoção de práticas alimentares (e de vida) saudáveis (Brasil, 2005a).

Uma alternativa de ação para a alimentação saudável deve favorecer, por exemplo, o deslocamento do consumo de alimentos pouco saudáveis para alimentos mais saudáveis. Supervalorizar ou mistificar determinados alimentos em função de suas características nutricionais ou funcionais também não deve constituir a prática da promoção da alimentação saudável. Alimentos nutricionalmente ricos devem ser valorizados e entrarão naturalmente na dieta adotada, sem que se precise mistificar uma ou mais de suas características, tendência esta muito explorada pela propaganda e publicidade de alimentos funcionais e complementos nutricionais.

De acordo com os princípios de uma alimentação saudável, todos os grupos de alimentos devem compor a dieta diária. A alimentação saudável deve fornecer água, carboidratos, proteínas, lipídios, vitaminas, fibras e minerais, os quais são insubstituíveis e indispensáveis ao bom funcionamento do organismo. A diversidade dietética que fundamenta o conceito de alimentação saudável pressupõe que nenhum alimento específico, ou grupo deles isoladamente, é suficiente para fornecer todos os nutrientes necessários a uma boa nutrição e conseqüente manutenção da saúde.

$\mathrm{O}$ ato da alimentação deve estar inserido no cotidiano das pessoas, como um evento agradável e de socialização. A estratégia para promoção da alimentação saudável também deve levar em consideração modificações históricas importantes como o crescente consumo de alimentos industrializados, pré-preparados ou prontos que respondem a uma demanda de praticidade.

Destacam-se algumas orientações do Guia (Brasil, 2005a) sobre as ações recomendadas ao governo e setor 
produtivo para a colocação em prática, as diretrizes:

Diretriz 1 - Os alimentos saudáveis e as refeições

- Garantir a qualidade dos alimentos - in natura e processados - colocados no mercado para consumo da população.

- Assegurar o cumprimento da legislação que promove o aleitamento materno enquanto direito da criança à alimentação adequada.

- Regulamentar estratégias de marketing de alimentos, em todas as formas de mídia, principalmente aquelas direcionadas para crianças e adolescentes.

Em relação aos compostos bioativos presentes nas verduras, legumes e ervas nativas do Brasil, o guia destaca que com base nos conhecimentos atualizados, a orientação permanece a mesma: "uma alimentação rica em frutas, legumes e verduras, fontes naturais de vitaminas, minerais e compostos bioativos, é fundamental para a manutenção da saúde" (Brasil, 2005a).

Os rótulos possuem uma grande quantidade de informação imprescindível ao consumidor. Um exemplo de alegação de propriedade funcional que pode constar nos rótulos dos alimentos, desde que previamente avaliada e aprovada pela Anvisa é: "As fibras alimentares auxiliam o funcionamento do intestino. Seu consumo deve estar associado a uma dieta equilibrada e a hábitos de vida saudáveis". Naturalmente a indústria dará maior destaque às características positivas de seu produto. Desta maneira, é importante analisar mais de uma informação. Por exemplo, um produto com alto teor de fibra - que é uma característica positiva, poderá, em contrapartida, ter alto teor de gordura, açúcar ou sódio. Outro produto com alto teor de cálcio pode ter elevada concentração de gordura saturada. Cada vez mais, é importante que o consumidor tenha acesso a informação, fortalecendo-o na capacidade de análise e decisão para optar por um ou outro produto, frente à indiscriminada quantidade de informações disponíveis nos diferentes veículos da mídia e publicidade (Brasil, 2005a).

\section{Diretriz Especial 1 - Atividade Física}

O princípio fundamental para manter um balanço energético é o equilíbrio entre ingestão e gasto energético. A atividade física pode ser definida como qualquer movimento realizado pelo sistema esquelético com gasto de energia. A atividade física adotada ao longo do curso da vida contribui para a prevenção e para a reversão de limitações funcionais. Isso é particularmente importante ao considerar-se o aumento da expectativa de vida no Brasil.

Mesmo na ausência de evidências conclusivas, estabeleceu-se que a transição de sobrepeso à obesidade pode ser prevenida com atividades de moderada intensidade por 45 a 60 minutos por dia (Brasil, 2005a). Uma das orientações sobre as ações recomendadas ao governo e setor produtivo é desenvolver formas de divulgação e comunicação social que informem e valorizem a adoção de modos de vida saudáveis, conjugando a promoção da alimentação saudável e a prática de atividade física regular.

\section{Política Nacional de Promoção da Saúde (Brasil, 2006)}

A Política Nacional de Promoção da Saúde tem como objetivo geral "promover a qualidade de vida e reduzir vulnerabilidade e riscos à saúde relacionados aos seus determinantes e condicionantes - modos de viver, condições de trabalho, habitação, ambiente, educação, lazer, cultura, acesso a bens e serviços essenciais".

A estratégia para implementação da Política Nacional de Promoção da Saúde relacionada com a alimentação é o estímulo à inserção de ações de Promoção da Saúde em todos os níveis de atenção, com ênfase na atenção básica, voltadas às ações de cuidado com o corpo e a saúde; alimentação saudável e prevenção e controle do tabagismo.

De acordo com Brasil (2006), compete ao gestor federal definir ações de promoção da saúde intersetoriais e pluriinstitucionais de abrangência nacional que possam impactar positivamente nos indicadores de saúde da população. Para o biênio 2006-2007 foram priorizadas as seguintes ações específicas voltadas para a alimentação saudável:

- Promover ações relativas à alimentação saudável visando ao cumprimento do direito humano à alimentação adequada.

- Promover articulação intra e intersetorial visando à implementação da Política Nacional de Promoção da Saúde por meio do reforço à implementação das diretrizes da Política Nacional de Alimentação e Nutrição e da Estratégia Global. Firmar uma agenda/pacto/compromisso social com diferentes setores (poder legislativo, setor produtivo, órgãos governamentais e não-governamentais, organismos internacionais, setor de comunicação e outros), definindo os compromissos e responsabilidades sociais de cada setor, com o objetivo de favorecer/garantir hábitos alimentares mais saudáveis na população, possibilitando a redução e o controle das taxas das doenças crônicas nãotransmissíveis - DCNT no Brasil. Articulação e mobilização intersetorial para a proposição e elaboração de medidas regulatórias que visem promover a alimentação saudável e reduzir o risco de DCNT, com es- 
pecial ênfase para a regulamentação da propaganda e publicidade de alimentos.

- Disseminar a cultura da alimentação saudável em consonância com os atributos e princípios do Guia Alimentar para a População Brasileira. Estimular ações de empoderamento do consumidor para o entendimento e uso prático da rotulagem geral e nutricional dos alimentos e monitorar o teor de sódio dos produtos processados, em parceria com a Anvisa e os órgãos da vigilância sanitária em Estados e Municípios.

\section{CONSIDERAÇÕES FINAIS}

Observa-se a convergência das políticas apresentadas no sentido de garantir informação fidedigna aos consumidores e fortalecer sua capacidade de entendimento dessas informações para que os mesmos possam fazer escolhas alimentares mais saudáveis. Também se reconhece que a alimentação saudável como medida de promoção da saúde não pode ser dissociada da adoção de hábitos de vida saudáveis, especialmente a prática de atividade física. Assim, as mensagens das alegações foram definidas e padronizadas pela Anvisa (2005a), condicionando o benefício alegado pelo consumo do alimento à adoção de uma dieta equilibrada e de hábitos de vida saudáveis. De acordo com as referidas políticas, os alimentos que suportam alegações não podem contribuir para o aumento de incidência de sobrepeso, obesidade e de outras doenças crônicas nãotransmissíveis. Desta forma não devem ser aprovadas alegações para alimentos que possam desestimular o aleitamento materno, ou que contenham elevada carga de energia, elevado conteúdo de açúcares livres, de sal, de gorduras saturadas e de gorduras trans. Além disso, as alegações devem ter sólida comprovação científica.

\section{AGRADECIMENTOS}

À Coordenação de Aperfeiçoamento de Pessoal de Nível Superior - CAPES.

\section{ABSTRACT \\ Health policies and functional property and health claims for food in Brazil}

This article establishes the relationship between public health policies in Brazil and the criteria adopted by Anvisa for the approval of functional property claims and, or, of health for food and for bioactive substances and isolated products. It also comments on Brazilian Legislation about functional property claimed food and, or, on healthy claimed food, new foods, bioactive substances and isolated probiotics, emphasizing the obligation to registering such products before the competent agencies. It presents the National Feeding and Nutrition Policies directives, National Policy for Health Promotion, Feeding Guide for the Brazilian Population and Global Strategy on Diet, Physical Activity and Health under the scope of World Health Organization. It enhances the convergence of directives of these policies that focus on the reduction of non-transmissible chronic diseases in the public by means of healthy feeding and physical activity. It highlights the orientations directed to governments and to the industry so as to implement these procedures. Through the presentation of evaluation criteria for the scientific basis of the claims, proposed by international agencies, it aims at offering subsidies for the understanding of the food technology over public health, and for the understanding of the criteria adopted by Anvisa for the evaluation of functional property claims and health.

UNITERMS: Function claims. Health claims. Functional foods. Legislation. Health policies.

\section{REFERÊNCIAS BIBLIOGRÁFICAS}

AGÊNCIA NACIONAL DE VIGILÂNCIA SANITÁRIA. ANVISA. (Brasil). Alimentos. Comissões e Grupos de Trabalho. Comissão Tecnocientífica de Assessoramento em Alimentos Funcionais e Novos Alimentos. Alimentos com Alegações de Propriedades Funcionais e ou de Saúde, Novos Alimentos/Ingredientes, Substâncias Bioativas e Probióticos. Atualizado em 11 de janeiro de 2005. VIII-Lista das Alegações Aprovadas. Disponível em: <http:www.anvisa.gov.br/alimentos/comissões/ tecno.htm>. Acesso em: 1 set. 2005. 2005a.

Gerência de Monitoração e Fiscalização da Propaganda, de Publicidade, de Promoção e de Informação de Produtos Sujeitos à Vigilância Sanitária (GPROP). Guia do Projeto de Monitoração de Propaganda e Publicidade de Produtos Sujeitos à Vigilância Sanitária. Brasília: 2004, 110 p. 2004a. (Versão Dez. 2004).

. Informe Técnico $\mathrm{n}^{\circ}$ 9, de 21 de maio de 2004, Orientação para utilização, em rótulos de alimentos, de alegação de propriedades funcionais de nutrientes com funções plenamente reconhecidas pela comunidade científica (item 3.3 da Resolução ANVS/MS nº18/99). Disponível em: $<$ http://www.anvisa.gov.br/alimentos/ informes/09_210504.htm>.Acesso em: 6abr. 2005. 2004b. 
Informe Técnico $\mathrm{n}^{\mathrm{o}}$ 19, de 29 de agosto de 2006. Assunto: Procedimentos para o enquadramento dos Cogumelos comestíveis em cápsulas, comprimidos e tabletes na área de alimentos. Disponível em: $<$ http:// www.anvisa.gov.br/alimentos/informes/19 300806.htm?. Acesso em: 5 set. 2006.

. Portaria $\mathrm{n}^{\circ} 15$, de 30 de abril de 1999. Institui junto à Câmara Técnica de Alimentos a Comissão de Assessoramento Tecnocientífico em Alimentos Funcionais e Novos Alimentos, com a incumbência de prestar consultoria e assessoramento em matéria relacionada a alimentos funcionais e novos alimentos, segurança de consumo e alegação de função em rótulos, submetidos por lei ao regime de vigilância sanitária. Diário Oficial da República Federativa do Brasil. Brasília, 03 maio.1999. Disponível em: $<$ http://e$1 \mathrm{e} \mathrm{g} \mathrm{i} \mathrm{s.bv} \mathrm{s.br/le} \mathrm{is} \mathrm{ref/pub} 1 \mathrm{i} \mathrm{c} /$ showAct.php?id=51\&mode=PRINT_VERSION $>$. Acesso em: 24 out. 2005. 1999a.

. Portaria $\mathrm{n}^{\circ} 386$, de 26 de setembro de 2005 . Altera a denominação e a composição da Comissão instituída pela Portaria n ${ }^{\circ} 15$ ANVISA, de 30 de abril de 1999 para Comissão de Assessoramento Tecnocientífico em Alimentos com Alegação de Propriedades Funcional e ou de Saúde e Novos Alimentos. Diário Oficial da República Federativa do Brasil. Brasília, 27 set. 2005. Disponível em: <http://e-legis.bvs.Br/leisref/public/ showAct.php?mode=PRINT_VERSION\&id=18884>. Acesso em: 28 out. 2005. 2005b.

Portaria $n^{\circ} 398$, de 30 de abril de 1999. Regulamento Técnico que Estabelece as Diretrizes Básicas para Análise e Comprovação de Propriedades Funcionais e ou de Saúde Alegadas em Rotulagem de Alimentos. Diário Oficial da República Federativa do Brasil. Brasília, 03 maio. 1999. Disponível em: <http://e-legis.bvs.br/leisref/ public/showAct.php?id=11297\& mode=PRINT_ VERSION>. Acesso em: 24 out.2005. 1999b.

. Resolução no 16, de 30 de abril de 1999. Aprova o Regulamento Técnico de Procedimentos para Registro de Alimentos e ou Novos Ingredientes. Diário Oficial da República Federativa do Brasil. Brasília, 03 dez. 1999. Disponível em: <http:// e-legis.bvs.br/leisref/public/ showAct.php?id=107>. Acesso em: 6 abr. 2005. 1999c.
Resolução no 17, de 30 de abril de 1999. Aprova o Regulamento Técnico que Estabelece as Diretrizes Básicas para Avaliação de Risco e Segurança dos Alimentos. Diário Oficial da República Federativa do Brasil. Brasília, 03 dez. 1999. Disponível em: <http://elegis.bvs.br/leisref/public/showAct.php?id=108>. Acesso em: 6 abr. 2005. 1999d.

Resolução $n^{\circ} 18$, de 30 de abril de 1999. Aprova o Regulamento Técnico que Estabelece as Diretrizes Básicas para Análise e Comprovação de Propriedades Funcionais e ou de Saúde Alegadas em Rotulagem de Alimentos. Diário Oficial da República Federativa do Brasil. Brasília,03 nov. 1999. Disponível em: <http://elegis.bvs.br/leisref/public/showAct.php?id=109>. Acesso em: 6 abr. 2005. 1999e.

. Resolução n 19, de 30 de abril de 1999. Aprova o Regulamento Técnico de Procedimentos para Registro de Alimento com Alegação de Propriedades Funcionais e ou de Saúde em sua Rotulagem. Diário Oficial da República Federativa do Brasil. Brasília, 10 dez. 1999. Disponível em: <http://e-legis.bvs.br/leisref/public/ showAct.php?id=110>. Acesso em: 30 ago. 2005. $1999 f$.

. Resolução n ${ }^{\circ} 22$, de 15 de março de 2000. Dispõe sobre os Procedimentos Básicos de Registro e Dispensa de Registro de Produtos Importados Pertinentes à Área de Alimentos. Diário Oficial da República Federativa do Brasil. Brasília, 16 mar. 2000. Disponível em: $<$ http://elegis.bvs.br/leisref/public/showAct.php?id=136>. Acesso em: 28 set. 2005. 2000a.

Resolução n ${ }^{\circ} 23$, de 15 de março de 2000. Dispõe sobre o Manual de Procedimentos Básicos para Registro e Dispensa da Obrigatoriedade de Registro de Produtos Pertinentes à Área de Alimentos. Diário Oficial da República Federativa do Brasil. Brasília, 07 fev. 2003. Disponível em: <http://www.engetecno.com.br/ resolucao 23_15_03_00.htm>. Acesso em: 28 nov. 2005. 2000 b.

Resolução RDC n 2, de 7 de janeiro de 2002. Aprova o Regulamento Técnico de Substâncias Bioativas e Probióticos Isolados com Alegação de Propriedades Funcionais e ou de Saúde. Diário Oficial da República Federativa do Brasil. Brasília, 9 jan. 2002. Disponível em: <http://e-legis.bvs.br/leisref/public/ showAct.php?id=9059 \&Word=>. Acesso em: 30 ago. 2005. 2002. 
Resolução RDC n 359, de 23 de dezembro de 2003. Aprova Regulamento Técnico de Porções de Alimentos Embalados para Fins de Rotulagem Nutricional. Diário Oficial da República Federativa do Brasil. Brasília, 26 dez. 2003. Disponível em: <http:/ /e-legis.anvisa.gov.br/leisref/public/ showAct.php?id $=9058 \&$ word $=>$. Acesso em: 5 set. 2006. 2003.

. Resolução RDC nº 278, de 22 de setembro de 2005. Aprova as categorias de Alimentos e Embalagens Dispensados e com Obrigatoriedade de Registro. Diário Oficial da República Federativa do Brasil. Brasília, 23 set. 2005. Disponível em: <http://e-legis.anvisa.gov.br/ leisref/public/showAct.php>. Acesso em: 27 abr. 2006. $2005 \mathrm{c}$.

AGGETT, P.J. et al. PASSCLAIM. Processo para a avaliação da base científica para alegações em alimentos. Consenso sobre critérios. Eur. J. Nutr., v. 44 (suppl. 1), p.5-36, 2005.

ARAI, S. Studies on functional foods in Japan - State of the art. Biosci., Biotechnol. Biochem., v.60, n.1, p.9-15, 1996.

ARAYA, H.; LUTZ, M.R. Alimentos funcionales y saludables. Rev. Chil. Nutr., v.30, n.1, p.8-14, 2003.

BRASIL. Decreto-Lei n ${ }^{\circ}$ 986, de 12 de outubro de 1969. Institui normas básicas sobre alimentos. Diário Oficial da União (D.O.U.), Brasília, 21 de outubro de 1969. Diário Oficial da República Federativa do Brasil. 1969. Brasília, Disponível em: <http://e-legis.bvs.br/leisref/ public/show Act.php>. Acesso em: 6 abr. 2005.

. Decreto n ${ }^{\circ} 3029$, de 16 de abril de 1999. (Versão Consolidada pela Procuradoria da ANVISA). Aprova o Regulamento da Agência Nacional de Vigilância Sanitária, e dá outras providências. Diário Oficial da República Federativa do Brasil. Brasília, 19 abr. 1999. Disponível em: <http://e-legis.bvs.br/leisref/public/ showAct.php?id+16605\&word=>. Acesso em: 19 set. 2005. 1999a.

. Decreto $\mathrm{n}^{\circ} 3571$, de 21 de agosto de 2000. Dá nova redação a dispositivos do Regulamento da Agência Nacional de Vigilância Sanitária, aprovado pelo Decreto $\mathrm{n}^{\circ} 3029$, de 16 de abril de 1999. Diário Oficial da República Federativa do Brasil. Brasília, 22 ago. 2000. Disponível em: <http://e-legis.bvs.br/leisref/public/ showAct.php?id+658\&word+decreto $>$. Acesso em: 10 nov. 2005.
Ministério da Saúde. Política Nacional de Promoção da Saúde. Anexo 1. 38 p. Disponível em: $<$ http://portal.saúde.gov.br/portal/arquivos/pdf/ portaria687-2006-anexo1.pdf $>$. Acesso em: 05 maio. 2006.

. Ministério da Saúde. Portaria $\mathrm{n}^{\circ} 710$, de 10 de junho de 1999. Aprova a Política Nacional de Alimentação e Nutrição. Diário Oficial da República Federativa do Brasil. Brasília, 11 jun. 1999. Disponível em: <http://elegis.anvisa.gov.br/leisref/public/ showAct.php?id=91\&word $=$. Acesso em: 06 out. 2006. 1999b.

. Ministério da Saúde. Secretaria de Atenção à Saúde. Coordenação-Geral da Política de Alimentação. Guia alimentar para a população brasileira. Brasília. Ministério da Saúde. Série A. Normas e Manuais Técnicos. 2005. Disponível em: <http:// www.materiasespeciais.com.br/ saude/guia/ guiaalimetar.doc $>$. Acesso em: 17 out. 2005. 2005a.

. Ministério da Saúde. Secretaria de Atenção à Saúde. Departamento de Atenção Básica. Política Nacional de Alimentação e Nutrição. Série B. Textos Básicos de Saúde. 2. ed. rev. Brasília, Ministério da Saúde, 2005, 48p. 2005 b.

. Ministério da Saúde. Secretaria de Vigilância Sanitária. SVS. Portaria n 27, de 13 de janeiro de 1998. Aprova o Regulamento Técnico referente à Informação Nutricional Complementar (declarações relacionadas ao conteúdo de nutrientes), constantes do anexo desta Portaria. Diário Oficial da República Federativa do Brasil. Brasília, 16 jan. 1998. Disponível em: <http://elegis.bvs.br/leisref/public/showAct.php?id+97\&word=>. Acesso em: 5 nov. 2005. 1998.

CARVALHO, J.E. Toxicidade pré-clínica: fitoterápicos e alimentos com propriedades funcionais ou de saúde. 2004. Disponível em: <http://www.abma.com.br/2004/ notes/2005.pdf >. Acesso em: 14 out. 2005.

. Functional Foods: Benefits, Concerns and Challenges - A Position Paper from the American Council on Science and Health. J. Nutrition v. 132, n.12, p. 3772-3781, 2002.

HASLER, C.M. Functional Foods: their role in disease prevention and health promotion. Food Technol., v.52, n.11, p.63-70, 1998. 


\section{INTERNATIONAL FOOD INFORMATION COUNCIL} FOUNDATION. IFIC. Alimentos Funcionales. 2004. Disponível em: <http://www.ific.org/sp/nutrition/ functional/index.cfm?render forprint $=1>$. Acesso em: 21 out. 2005 .

LAJOLO, F.M. Functional foods: Latin American Perspectives. Br. J. Nut., v.88, Suppl. 2, S145-S150, 2002.

. Introdução Alimentos Funcionais. In: I Congresso Internacional sobre Alimentos Funcionais - Ciência, Inovação e Regulamentação. FIESP. 24 de abril. São Paulo. 2006. Disponível em: $<$ http://www.fiesp.com.br/ alimentosfuncionais $>$. Acesso em: 05 maio. 2006.

OLIVEIRA, T.P. A aplicação da Política Nacional de Alimentação e Nutrição. In: I Congresso Internacional Sobre Alimentos Funcionais - Ciência, Inovação e Regulamentação. Apresentação Power Point. São Paulo. abril. 2006. Disponível em: <http://www.fiesp.com.br/ alimentos funcionais $>$. Acesso em: 05 maio. 2006.

SANTOS, C.F. Panorama Brasil - Fluxo de aprovação das alegações. In: I Congresso Internacional sobre Alimentos Funcionais - Ciência, Inovação e Regulamentação. FIESP. 24 de abril. São Paulo. 2006. Disponível em: $<$ http:/ /www.fiesp.com.br/alimentosfuncionais $>$. Acesso em: 05 maio. 2006.
WORLD HEALTH ORGANIZATION .WHO. Global strategy on diet, physical activity and health: fiftyseventh World Health Assembly Wha 57.17. 22 May 2004. Disponível em:<www.who.int>. Acesso em: 10 jul. 2005.

. Macroeconomics and health: investing in health for economic development. Geneva. 213p. 2001. Disponível em: <http://www.emro.who.int/cbi/pdf/CMHReport HQ.pdf >. Acesso em: 10 jul. 2006.

. The world health report 2002. Reducing risks, promoting healthy life. Geneva. Disponível em: $<$ http:/ /www.who.int/whr/2002/en/index.html $>$. Acesso em: 10 jul. 2006.

. Diet, Nutrition and the Prevention of Chronic Diseases. Report of a joint WHO/FAO expert consultation. (WHO Technical Report Series, 916).Geneva. 149 p. 2003. Disponível em: $<$ http:// whqlibdoc.who.int/trs/who_TRS_916.pdf $>$.Acesso em: 31 maio. 2006.

Recebido para publicação em 07 de dezembro de 2006. Aceito para publicação em 23 de abril de 2007. 